\title{
Determinants of Voltage Attenuation in Neocortical Pyramidal Neuron Dendrites
}

\author{
Greg Stuart ${ }^{1,3}$ and Nelson Spruston ${ }^{2,3}$ \\ ${ }^{1}$ Division of Neuroscience, John Curtin School of Medical Research, Australian National University, Canberra, A.C.T. \\ 0200, Australia, ${ }^{2}$ Department of Neurobiology and Physiology, Institute for Neuroscience, Northwestern University, \\ Evanston, Illinois 60208-3520, and ${ }^{3}$ Max Planck Institut für Medizinische Forschung, Abteilung Zellphysiologie, \\ Heidelberg, 69120, Germany
}

How effectively synaptic and regenerative potentials propagate within neurons depends critically on the membrane properties and intracellular resistivity of the dendritic tree. These properties therefore are important determinants of neuronal function. Here we use simultaneous whole-cell patch-pipette recordings from the soma and apical dendrite of neocortical layer 5 pyramidal neurons to directly measure voltage attenuation in cortical neurons. When combined with morphologically realistic compartmental models of the same cells, the data suggest that the intracellular resistivity of neocortical pyramidal neurons is relatively low ( $\sim 70$ to $100 \Omega \mathrm{cm}$ ), but that voltage attenuation is substantial because of nonuniformly distributed resting con- ductances present at a higher density in the distal apical dendrites. These conductances, which were largely blocked by bath application of $\mathrm{CsCl}(5 \mathrm{~mm})$, significantly increased steadystate voltage attenuation and decreased EPSP integral and peak in a manner that depended on the location of the synapse. Together these findings suggest that nonuniformly distributed Cs-sensitive and -insensitive resting conductances generate a "leaky" apical dendrite, which differentially influences the integration of spatially segregated synaptic inputs.

Key words: voltage attenuation; dendrite; intracellular resistivity; neocortical pyramidal neuron; hyperpolarization-activated conductance; I $I_{h}$; sag; cesium
Synaptic and regenerative potentials generated in the dendritic tree propagate toward the soma and axon, where they may result in action potential initiation if threshold is reached (Stuart et al., 1997b). The manner in which these potentials propagate from the dendrites to the soma depends on a number of factors, including passive properties such as the membrane resistivity $\left(R_{\mathrm{m}}\right)$, the membrane capacitance $\left(C_{\mathrm{m}}\right)$, and intracellular resistivity $\left(R_{\mathrm{i}}\right)$, as well as the active properties of the dendritic membrane. The vast majority of previous studies of the passive membrane properties of the dendritic trees of CNS neurons, however, have inferred these properties from somatic microelectrode or patch-pipette recordings (Coombs et al., 1959; Rall, 1959; Lux et al., 1970; Iansek and Redman, 1973; Barrett and Crill, 1974; Brown et al., 1981; Durand et al., 1983; Clements and Redman, 1989; Stratford et al., 1989; Spruston and Johnston, 1992; Major et al., 1994; Rapp et al., 1994; Thurbon et al., 1994; Bekkers and Stevens, 1996; Thurbon et al., 1998). Two notable exceptions include Shelton (1985), who based his analysis on somatic and dendritic microelectrode recordings from cerebellar Purkinje cells by Llinas and Sugimori (1980a,b), and Meyer and coworkers (1997), who used voltage-sensitive dyes and imaging techniques to estimate the

Received Oct. 17, 1997; revised Feb. 18, 1998; accepted Feb. 25, 1998.

We thank M. Häusser for helpful discussions, S. Redman for comments on this manuscript, A. Borst and J. Haag for assistance with morphological reconstructions, M. Hines for assistance and modifications to NEURON, and M. Migliore for help with the model of $I_{\mathrm{h}}$. This work was supported by a Human Frontiers Program Grant to G.S. and N.S., the National Health and Medical Research Council and Australian Research Council of Australia (G.S.), and National Institutes of Health (NS35180) and the Sloan Foundation (N.S.).

Correspondence should be addressed to Dr. Greg Stuart, Division of Neuroscience, John Curtin School of Medical Research, Australian National University, Canberra, A.C.T. 0200, Australia.

Copyright $\odot 1998$ Society for Neuroscience $\quad 0270-6474 / 98 / 183501-10 \$ 05.00 / 0$ dendritic properties of hippocampal pyramidal neurons in culture.

Clearly, the electrical properties of the dendritic tree will be determined with greater accuracy if electrical recordings are made directly from the dendrites themselves, preferably using the patch-clamp technique to avoid the electrical leak around the electrode generated by microelectrode recordings. The use of infrared differential interference contrast microscopy now makes this possible (Stuart et al., 1993). Moreover, this method allows recordings to be made from more than one site on the same neuron (Stuart and Sakmann, 1994), allowing direct measurement of voltage attenuation between different locations. Here we used simultaneous patch-pipette recordings from the soma and apical dendrite of neocortical neurons in brain slices to measure voltage attenuation along the apical dendrites of layer 5 pyramidal neurons. These recordings were then combined with morphologically realistic compartmental models of the same cells, to provide the first estimates of $R_{\mathrm{i}}$ based on direct measurement of voltage attenuation in neurons. The results suggest that $R_{\mathrm{i}}$ is lower than estimated in recent modeling studies (Shelton, 1985; Stratford et al., 1989; Fromherz and Muller, 1994; Major et al., 1994; Rapp et al., 1994; Thurbon et al., 1994; Bekkers and Stevens, 1996) (however, see Thurbon et al., 1998). Furthermore, simulations of the experimental data suggest that multiple resting conductances are nonuniformly distributed, generating a "leaky" apical dendrite, which increases the amount of steady-state voltage attenuation and influences synaptic integration by decreasing EPSP amplitude and duration.

\section{MATERIALS AND METHODS}

Slice preparation and recording. Slices $(300 \mu \mathrm{m})$ were prepared from somatosensory cortex of 3-week-old Wistar rats using standard techniques and visualized using a Zeiss Axioskop microscope equipped with 
a $40 \times$ water immersion lens. Both the soma and dendrites of layer 5 pyramidal neurons were visualized using infrared differential interference contrast (IR-DIC) optics. Patch-pipette recordings from the soma and apical dendrite (50-560 $\mu \mathrm{m}$ from the soma) were obtained as described previously (Stuart et al., 1993). During recordings, slices were perfused with artificial CSF of the following composition: $125 \mathrm{~mm} \mathrm{NaCl}$, $25 \mathrm{~mm} \mathrm{NaHCO}_{3}, 25 \mathrm{~mm}$ glucose, $2.5 \mathrm{~mm} \mathrm{KCl}, 1.25 \mathrm{~mm} \mathrm{NaH}_{2} \mathrm{PO}_{4}, 2 \mathrm{~mm}$ $\mathrm{CaCl}_{2}$, and $1 \mathrm{mM} \mathrm{MgCl}_{2}$, pH 7.4, with $95 \% \mathrm{O}_{2}$ and $5 \% \mathrm{CO}_{2}$, and maintained at $35-37^{\circ} \mathrm{C}$. For whole-cell recordings, patch pipettes $(4-7$ $\mathrm{M} \Omega$ for somatic; 8-10 $\mathrm{M} \Omega$ for dendritic) were filled with a potassium gluconate-based solution (120 mM potassium gluconate, $20 \mathrm{mM} \mathrm{KCl,} 10$ mM HEPES, $10 \mathrm{~mm}$ EGTA, $2 \mathrm{~mm} \mathrm{Na}_{2}$-ATP, and $2 \mathrm{mM} \mathrm{MgCl}_{2}, \mathrm{pH} 7.3$ with $\mathrm{KOH})$ to which biocytin $(0.5 \%)$ was added. Series resistances ranged from 5 to $17 \mathrm{M} \Omega$ at the soma and from 10 to $90 \mathrm{M} \Omega$ at the dendritic recording site. During perforated-patch recordings, pipettes tips were filled with $150 \mathrm{~mm} \mathrm{KCl}$ plus $10 \mathrm{~mm} \mathrm{HEPES}, \mathrm{pH} 7.4$ with $\mathrm{KOH}$, and then back-filled with the same solution into which was dissolved gramicidin (9 $\mu \mathrm{g} / \mathrm{ml}$; Sigma, St. Louis, MO) (Kyrozis and Reichling, 1995) plus Lucifer yellow (1 mg/ml; Sigma). Perforation was detected by a slow reduction in series resistance to 30-65 $\mathrm{M} \Omega$. At the end of the experiment, fluorescence microscopy was used to determine whether the recorded cell was stained with Lucifer yellow (indicating rupture of the membrane patch). If staining was detected the data were discarded.

Somatic and dendritic patch-pipette recordings (seal resistances $>1$ $\mathrm{G} \Omega$ ) were made with identical amplifiers (Axoclamp, Axon Instruments, Foster City, CA), and bridge balance and capacitance compensation were performed for both recordings. Hyperpolarizing somatic long (200 msec, $-50 \mathrm{pA})$ and short $(1 \mathrm{msec}, 2 \mathrm{nA})$ current pulses were applied from the resting membrane potential (soma: -61 to $-69 \mathrm{mV}$; dendrite: -57 to $-64 \mathrm{mV}$ ) under control conditions and after application of $5 \mathrm{mM} \mathrm{CsCl}$ to the bath. Because the accuracy of the measured steady-state voltage attenuation depends on correct bridge balance of the somatic electrode (through which current was injected), several control double somatic recordings were obtained to confirm this. In six such recordings the average bridge balance error was only $4.0 \pm 2.0 \%$. Furthermore, somatic input resistance estimates obtained from long and short pulses were in agreement (Durand et al., 1983), also suggesting that the somatic pipette resistance was adequately compensated. Double somatic recordings were also used to compare the time course of the somatic short current pulse response detected by the current passing and noncurrent passing pipettes in external $\mathrm{CsCl}$. These experiments showed that $2 \mathrm{msec}$ after the termination of the short current pulse the voltage response detected by the current passing pipette was identical to that detected by the noncurrent passing pipette $(n=6)$. This finding was independent of bridge balance errors or inadequate electrode compensation of the current passing pipette. As a consequence, fits to the somatic short current pulse data were made to the response $2 \mathrm{msec}$ after termination of the short current pulse, whereas for dendritic short current pulse data the whole response was fit. Extracellular synaptic stimulation was performed with a patch pipette filled with oxygenated extracellular solution whose tip (diameter $2 \mu \mathrm{m}$ ) was placed within $20 \mu \mathrm{m}$ of the dendritic recording pipette. All experiments, except those in which EPSPs were evoked by extracellular stimulation, were performed in the presence of 6-cyano-7-nitroquinoxaline-2,3-dione (10 $\mu \mathrm{M}$; Tocris), DL-2-amino-5phosphonopentanoic acid (50 $\mu \mathrm{M}$; Tocris), and bicuculline methoiodide (20 $\mu \mathrm{M}$; Sigma) to block spontaneous excitatory and inhibitory synaptic events. Responses shown are averages of between 20 and 130 sweeps.

Data analysis. Steady-state attenuation and input resistance measurements were made over the last $20 \mathrm{msec}$ of long $200 \mathrm{msec}$ hyperpolarizing current pulses. It was impossible to accurately measure the membrane time constant under control conditions because of the presence of the hyperpolarization-activated conductance $I_{\mathrm{h}}$ (see Results). An estimate of the membrane time constants in control was obtained, however, from single exponential fits to the initial somatic response during long somatic current pulses (over approximately the first $30 \mathrm{msec}$ ). In the presence of $\mathrm{CsCl}$ used to block $I_{\mathrm{h}}$, the apparent membrane time constant was determined by the slowest time constant obtained from multi-exponential fits (at least three) to both the somatic and dendritic voltage responses during somatic long and short current pulses (average value was used). Pooled data are expressed as mean \pm SEM, and tests for statistical difference used an unpaired $t$ test at a significance level of 0.05 . Mean squared error (MSE) was calculated on a point by point basis, where at each point the difference between the response generated by a particular model and the recorded experimental data were determined and squared, and the average value was calculated for all data points fitted.
Neuron staining and morphology. All cells were filled during whole-cell recording with biocytin $(0.5 \%)$ contained in both somatic and dendritic patch pipettes and IR-DIC images made of the soma and the main apical dendrite to beyond the dendritic recording site. At the end of the experiment, both pipettes were withdrawn to form outside-out patches, thus resealing the membrane. In addition, after termination of each experiment the dendritic recording electrode was moved laterally $\sim 100$ $200 \mu \mathrm{m}$ and then "crammed" into the slice. Slices were then fixed and subsequently stained using an avidin-horseradish peroxide reaction (Vector Laboratories, Burlingame, CA). Slices were not dehydrated after fixation to minimize tissue shrinkage, were not resliced, and were mounted in an aqueous mounting medium. Comparison of somatic and apical dendritic diameters from IR-DIC images of the living cell with that of the cell after fixation and staining allowed us to directly determine the extent of tissue shrinkage, which was found to be minimal. As a consequence, no corrections were made for tissue shrinkage. The site of dendritic recording was identified by comparison of the filled cell with the IR-DIC image taken during recording, and by identification of the indentation left in the slice by the dendritic recording electrode. In addition, in the reconstructed and modeled neurons the site of dendritic recording was easily identifiable because it was in all cases at, or close to, the main apical branch point.

Morphological reconstruction. Three cells were reconstructed using a semiautomated procedure. The stained and fixed slices were placed on a microscope slide that was mounted on a motorized, three-dimensional micromanipulator (Luigs and Neumann) connected to a personal computer. The neuron was imaged with a video camera (C2400-07, Hammamatsu) using infrared light, and the image was viewed on a video monitor using a Zeiss $40 \times(0.75$ numerical aperture $)$ water immersion lens with $4 \times$ extra magnification. The slide was moved to each reconstructed point on the neuron, and the diameter at that point was measured using an Argus-10 system (Hamamatsu). Using this method we estimate that we were able to measure dendritic diameters to within $\sim 0.2$ $\mu \mathrm{m}$. The branching state and the displayed diameter were entered into a computer program (Jürgen Haag, MPI) that recorded these data along with the three-dimensional coordinates read from the motorized micromanipulator. The three neurons that were reconstructed consisted of 1099,1112 , and 610 compartments corresponding to the same number of reconstructed points.

Neuronal modeling. The three-dimensional coordinates and their corresponding diameters and branching pattern were converted to a format readable by the neuronal simulation program NEURON (Hines and Carnevale, 1997) using the program NTSCABLE (file://ftp.cnl.salk.edu/ pub/alain/ntscable_2.0.doc; J. C. Wathey, Salk Institute, San Diego, CA). All modeling was performed using NEURON (http://neuron.duke.edu; M. Hines, Yale University, New Haven, CT). Direct fitting was also done with NEURON, which calls the program PRAXIS (http:// performance.netlib.org/opt/praxis; Stanford Linear Accelerator Center, $3 / 1 / 73$ ) for parameter searching using the principal axis method (Brent, 1973). Minimization of the MSE was used to determine the model that gave the best fit. Spines were incorporated into each model on all apical dendritic compartments beyond $100 \mu \mathrm{m}$ from the soma on the apical dendritic tree and beyond $20 \mu \mathrm{m}$ from the soma on basal dendrite compartments. Our own estimates of spine density agreed with published values (Larkman, 1991). Spines were not modeled explicitly, but their effects on membrane area were modeled by decreasing $R_{\mathrm{m}}$ and increasing $C_{\mathrm{m}}$ by a factor of 2 in spiny compartments (Shelton, 1985; Holmes, 1989; Larkman, 1991). In a few cases cells were filled with Lucifer yellow (Sigma), and three-dimensional reconstruction using confocal microscopy in the living slice was used to asses whether the cross sections of the soma and dendrites were cylindrical, as is assumed to be the case in NEURON. The cross section of dendrites, at least of the main apical dendrite $50 \mu \mathrm{m}$ from the soma, was found to be cylindrical; however, the cross section of the soma was ellipsoid. As a consequence, the diameters of somatic compartments were multiplied by 0.77 to make them equivalent to an ellipse $(\mathrm{a}=2 \mathrm{~b})$. In models in which $R_{\mathrm{i}}$ was determined simply from the amount of steady-state attenuation in $\mathrm{CsCl}$, we assumed that errors in our spine estimate or other morphological features that could not be discerned at the light microscopic level would contribute to errors in the surface area of the reconstructed cell. These effects were accounted for by adding a free parameter to the model ("areascale") that scaled the area of all compartments in the model in a way similar to the spine scale parameter (Bush and Sejnowski, 1993). The values of $C_{\mathrm{m}}$ and $R_{\mathrm{m}}$, respectively, in these models were obtained by multiplying the starting value of $C_{\mathrm{m}}\left(1 \mu \mathrm{F} / \mathrm{cm}^{2}\right)$ and dividing the starting value of $R_{\mathrm{m}}$ by 
the value of areascale. The areascale values used in the final uniform $R_{\mathrm{m}}$ models determined from the amount of steady-state attenuation in $\mathrm{CsCl}$ ranged from 0.9 to 1.3 . With the direct fitting approach, the areascale variable was eliminated, and both $R_{\mathrm{m}}$ and $C_{\mathrm{m}}$ were free parameters. The reversal potential of the resting leak was set to the recorded resting membrane potential measured in $\mathrm{CsCl}$ for each cell, which ranged from -78 to $-75 \mathrm{mV}$. Excitatory synaptic conductances were modeled by the sum of two exponentials $\left(\tau_{\text {rise }}=0.2 \mathrm{msec}, \tau_{\text {decay }}=3 \mathrm{msec}\right.$ ) with a reversal potential of $0 \mathrm{mV}$, and they were placed directly onto the indicated dendritic compartments.

$R_{\mathrm{m}}$ was either made uniform (i.e., the same in all compartments of the model) or modeled with the following nonuniform, sigmoidal function:

$$
R_{\mathrm{m}}=R_{\mathrm{m}}(\text { end })+\frac{R_{\mathrm{m}}(\text { soma })-R_{\mathrm{m}}(\text { end })}{1+e^{\left(d_{\text {half }}-d i s\right) / \text { steep }}}
$$

where $d_{\text {half }}$ is the distance at which the function is halfway between $R_{\mathrm{m}}$ (soma) and $R_{\mathrm{m}}$ (end), and steep determines the steepness of the decay from $R_{\mathrm{m}}$ (soma) to $R_{\mathrm{m}}$ (end) with distance (dis) from the soma to all dendritic compartments, including the basal and apical oblique dendrites. In nonuniform $R_{\mathrm{m}}$ models the final values of $R_{\mathrm{m}}$ (soma), $R_{\mathrm{m}}(\mathrm{end}), d_{\text {half }}$, $C_{\mathrm{m}}$, and $R_{\mathrm{i}}$ were optimized using the direct fitting routine in NEURON, with steep fixed at $50 \mu \mathrm{m}$. Steep was fixed at $50 \mu \mathrm{m}$ in these models because direct fitting using a range of steep values showed that steeper nonuniform functions of $R_{\mathrm{m}}$ generally gave better fits; however, as steep was reduced to $<50 \mu \mathrm{m}$ the improvement to the fit was insignificantly small.

In models that also included the hyperpolarization-activated conductance $\left(I_{\mathrm{h}}\right)$, this was modeled with a reversal potential of $-43 \mathrm{mV}$, which was calculated from the concentrations of $\mathrm{Na}$ and $\mathrm{K}$ in our solutions and the published permeability ratio $\left(P_{\mathrm{Na}} / P_{\mathrm{K}}\right)$ for $I_{\mathrm{h}}$ of 0.4 (Solomon and Nerbonne, 1993a). The voltage dependence and kinetics were also based on published data (Solomon and Nerbonne, 1993a,b), using a $Q_{10}$ of 3. Incorporation of a parameter, $R$, was used to scale the kinetics of $I_{\mathrm{h}}$ to optimize the fit to the control somatic long-pulse data. The density of the $I_{\mathrm{h}}$ conductance in individual compartments $\left(g_{\mathrm{h}}\right)$ was either made uniform or modeled with a similar sigmoidal function as in nonuniform $R_{\mathrm{m}}$ models, where:

$$
g_{\mathrm{h}}=g_{\mathrm{h}}(\text { soma })+\frac{g_{\mathrm{h}}(\mathrm{end})-g_{\mathrm{h}}(\text { soma })}{1+e^{\left(d_{\text {half }}-d i s\right) / \text { steep }}}
$$

and $g_{\mathrm{h}}$ (soma), $g_{\mathrm{h}}$ (end), $d_{\text {half }}$, dis, and steep have meanings similar to $R_{\mathrm{m}}$ (soma), $R_{\mathrm{m}}$ (end), $d_{\text {half }}$, dis, and steep as described in Equation 1 . The final values of $g_{\mathrm{h}}$ and $R$ for uniform $I_{\mathrm{h}}$ models, or $g_{\mathrm{h}}(\mathrm{soma}), g_{\mathrm{h}}(\mathrm{end}), d_{\text {half }}$, and $R$ for nonuniform $I_{\mathrm{h}}$ models, were optimized using the direct fitting routine in NEURON, with steep fixed at $50 \mu \mathrm{m}$ in nonuniform $I_{\mathrm{h}}$ models. As with nonuniform $R_{\mathrm{m}}$ models, direct fitting of nonuniform $I_{\mathrm{h}}$ models using a range of steep values showed that as steep was reduced to $<50$ $\mu \mathrm{m}$ the improvement to the fit was insignificantly small.

\section{RESULTS}

\section{Uniform models}

The attenuation of membrane voltage along the apical dendrite of neocortical layer 5 pyramidal neurons was directly measured during simultaneous somatic and dendritic whole-cell recordings in brain slices (Fig. $1 A-C$ ). Long ( $250 \mathrm{msec},-50 \mathrm{pA}$ ) and short (1 msec, $-2 \mathrm{nA})$ hyperpolarizing current pulses were injected through the somatic pipette, and the resulting voltage change was recorded simultaneously with the somatic and dendrite recording pipettes. Voltage responses to somatic long current pulses showed significant steady-state attenuation (Fig. 1D) and exhibited a sag (slow, partial repolarization), mediated by the hyperpolarizationactivated conductance $I_{\mathrm{h}}$ (Spain et al., 1987; Solomon and Nerbonne, 1993a). Bath application of $\mathrm{CsCl}$ (5 mM) blocked the sag in the somatic and dendritic current pulse voltage responses and caused a significant decrease in steady-state voltage attenuation (Fig. $1 E$; compare with $1 D$ ). Plots of steady-state attenuation versus the distance the dendritic recording was made from the soma showed that $50 \%$ steady-state attenuation occurred at a distance of $\sim 332 \mu \mathrm{m}$ from the soma in control conditions and 526 $\mu \mathrm{m}$ in $\mathrm{CsCl}$ (Fig. $1 F)(n=13)$. These finding show that Cssensitive conductances, such as $I_{\mathrm{h}}$, are on at rest and contribute significantly to steady-state voltage attenuation in the apical dendrites of neocortical layer 5 pyramidal neurons.

To check whether dialysis by the whole-cell pipette solution had an effect on the measured passive or active properties, a number of somatic perforated-patch recordings were made under control conditions and in the presence of $5 \mathrm{~mm}$ external $\mathrm{CsCl}$. Average somatic input resistance in control measured with whole-cell recording was $32.1 \pm 1.2 \mathrm{M} \Omega(n=13)$. This was not significantly different from that measured with perforated-patch recording $(34.6 \pm 4.4 \mathrm{M} \Omega ; n=5)$. In addition, the extent of sag in the somatic response to a long current pulse (control steady-state response/control peak response) was also not significantly different when measured with the different recording conditions $[0.81 \pm 0.01(n=13)$ with whole-cell compared with $0.86 \pm 0.03$ $(n=5)$ with perforated-patch]. These findings suggest that there was no significant effect of whole-cell recording on input resistance or $I_{\mathrm{h}}$. Average somatic input resistance in $\mathrm{CsCl}$ with wholecell recording $(51.7 \pm 1.9 \mathrm{M} \Omega ; n=13)$ was also not significantly different from that measured with perforated-patch recording $(56.4 \pm 9.2 \mathrm{M} \Omega ; n=5)$, showing that application of $\mathrm{CsCl}$ blocked resting conductances to a similar extent under the two recording conditions. Together these data suggest that the use of whole-cell recording had no significant effect on the basic passive or active electrophysiological parameters of neocortical layer 5 pyramidal cells examined in this study.

Full morphological reconstructions were obtained for three cells, in which dendritic recordings were obtained 508-563 $\mu \mathrm{m}$ from the soma (Fig. 1), and passive compartmental models of these cells were used to determine the value of the intracellular resistivity $\left(R_{\mathrm{i}}\right)$ consistent with the observed steady-state voltage attenuation. Initial values of the passive membrane resistivity $\left(R_{\mathrm{m}}\right)$ for each cell were obtained from the apparent membrane time constant $\left(\tau_{\mathrm{m}}\right)$ using the equation $\tau_{\mathrm{m}}=R_{\mathrm{m}} \cdot C_{\mathrm{m}}$, assuming $C_{\mathrm{m}}$ $=1 \mu \mathrm{F} / \mathrm{cm}^{2}$. Under control conditions, $\tau_{\mathrm{m}}$ was estimated to be $12.4 \pm 0.5 \mathrm{msec}(n=12)$, whereas in the presence of $5 \mathrm{~mm} \mathrm{CsCl}$ $\tau_{\mathrm{m}}$ ranged from 14 to $25 \mathrm{msec}$, with an average value of $20.9 \pm 0.8$ msec $(n=12)$. To account for uncertainty in the assumption that $C_{\mathrm{m}}=1 \mu \mathrm{F} / \mathrm{cm}^{2}$ (Brown et al., 1981; Shelton, 1985; Major et al., 1994), as well as uncertainty in the total surface area of the reconstructed neurons (caused by imperfect reconstruction of membrane invaginations or incorrect correction for spines; see Materials and Methods), a free parameter (defined as areascale) was used to scale $R_{\mathrm{m}}$ and $C_{\mathrm{m}}$ in each compartment while keeping their product equal to $\tau_{\mathrm{m}}$ (Bush and Sejnowski, 1993).

For each model, $R_{\mathrm{i}}$ was varied over the range 50 to $400 \Omega \mathrm{cm}$, and the areascale variable was adjusted to yield the measured somatic input resistance. This generated a family of models all with the same somatic input resistance and $\tau_{\mathrm{m}}$ but with different $R_{\mathrm{i}}$ values and different degrees of soma to dendrite steady-state attenuation. For each $R_{\mathrm{i}}$ value, steady-state attenuation ( $V_{\text {dendrite }}$ $V_{\text {soma }}$ ) was plotted against the corresponding $R_{\mathrm{i}}$ value (Fig. $2 A$, filled circles). The $R_{\mathrm{i}}$ value for a particular cell was then estimated by simply finding which value gave the correct amount of steadystate voltage attenuation (Fig. $2 A$ ). In the three cells reconstructed and modeled (Fig. $1 A-C$ ) the values of $R_{\mathrm{i}}$ that gave the observed steady-state attenuation were 151,147 , and $175 \Omega \mathrm{cm}$. These values also corresponded to the minimum observed mean squared error of the simulated responses to long current pulses (Fig. 2A, open circles) obtained using this initial, simple method. 
A

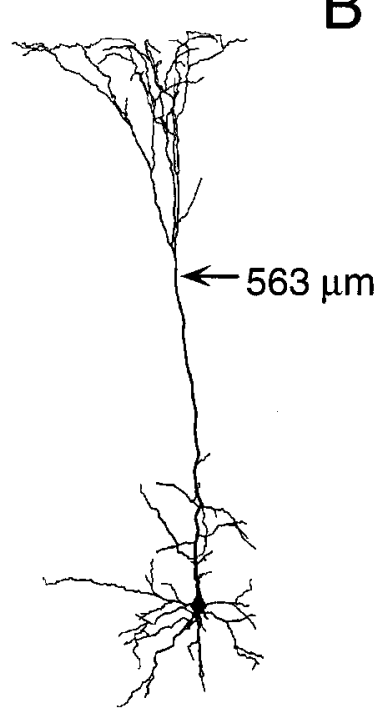

B

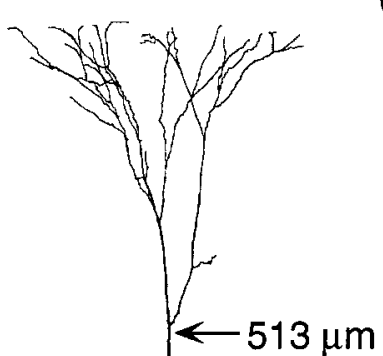

C
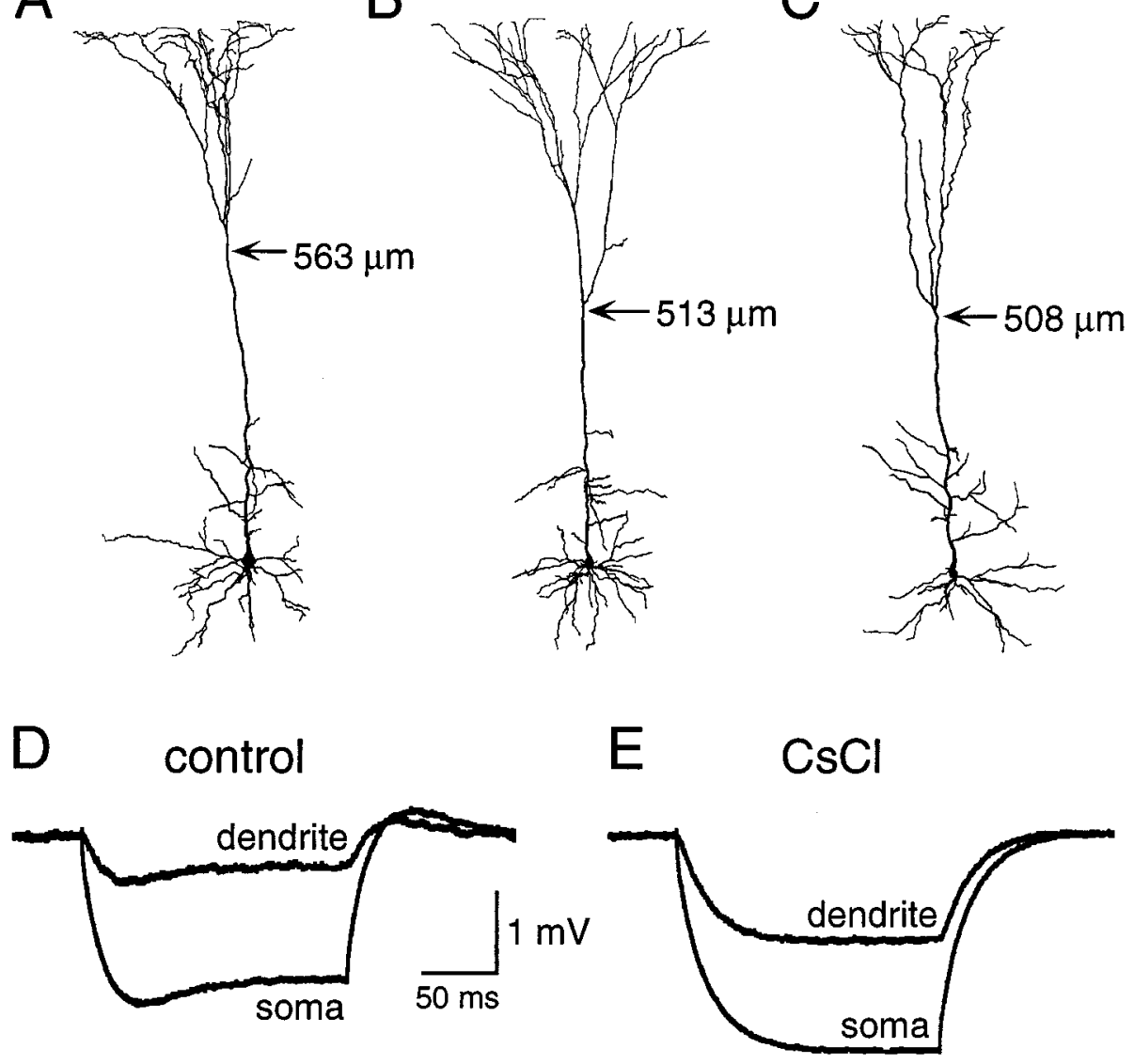

Figure 1. Experimental measurements of steady-state voltage attenuation in neocortical layer V pyramidal neurons. $A-C$, Representation of the dendritic morphology of three fully reconstructed cells, with the site of the dendritic recording indicated (arrow). The images shown were generated by NEURON using the measured coordinates and diameters for each cell (1099, 1112, and 610 compartments). $D-E$, Average somatic and dendritic responses obtained from the cell shown in $A$ during somatic $200 \mathrm{msec},-50 \mathrm{pA}$ current pulses in control $(D)$ and in the presence of $5 \mathrm{~mm} \mathrm{CsCl}(E) . F$, Steady-state voltage attenuation in control conditions $(\bigcirc)$ and in $5 \mathrm{~mm} \mathrm{CsCl}(\bullet)$ during dendritic recordings at different distances from the somata of 13 neurons. The smooth line is the best fit to the control (bottom line) and $\mathrm{CsCl}$ (top line) data arbitrarily fitted with a Gaussian function. The dotted lines indicate $50 \%$ steady-state attenuation, and the arrows indicate the distance from the soma where this occurred for recordings in control and $\mathrm{CsCl}$.

F

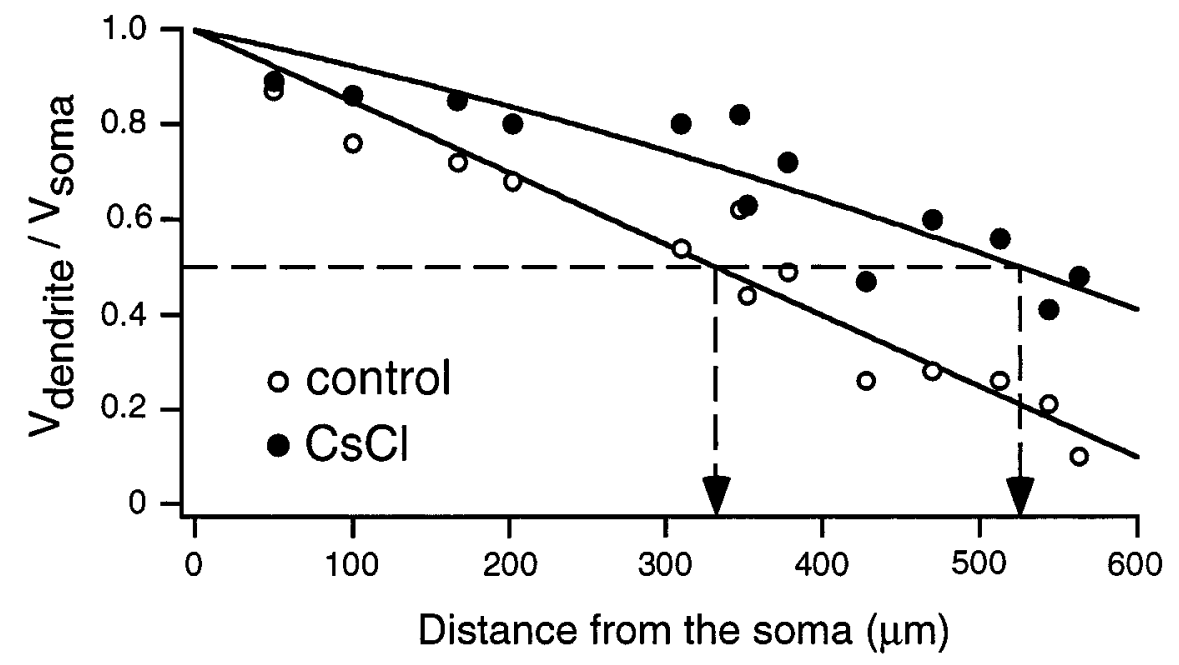

An overlay of the predicted response with the experimental data during long somatic current pulses for the cell depicted in Figure $1 A$ is shown in Figure $2 B$. Comparison of these simulations (broken lines) with the experimental data (solid lines) shows that although the model predicted accurately the amount of steady-state attenuation, the fit to the long-pulse data suffered from the problem that, compared with the experimental data, the predicted somatic voltage decayed too rapidly, whereas the predicted dendritic voltage decayed too slowly. Similar results were found in all three cells modeled. For each cell, the model parameters that best fit the steady-state attenuation in $\mathrm{CsCl}$ were then used to simulate the somatic and dendritic responses to somatic short current pulses in $\mathrm{CsCl}$ (Fig. 2C). Comparison of these simulations with the experimental data from each cell showed that, like the steady-state voltage responses, in all three cells modeled the fit to the short-pulse data suffered from the problem that the simulated somatic voltage decayed too rapidly, whereas the simulated dendritic voltage decayed too slowly (Fig. 2C). 

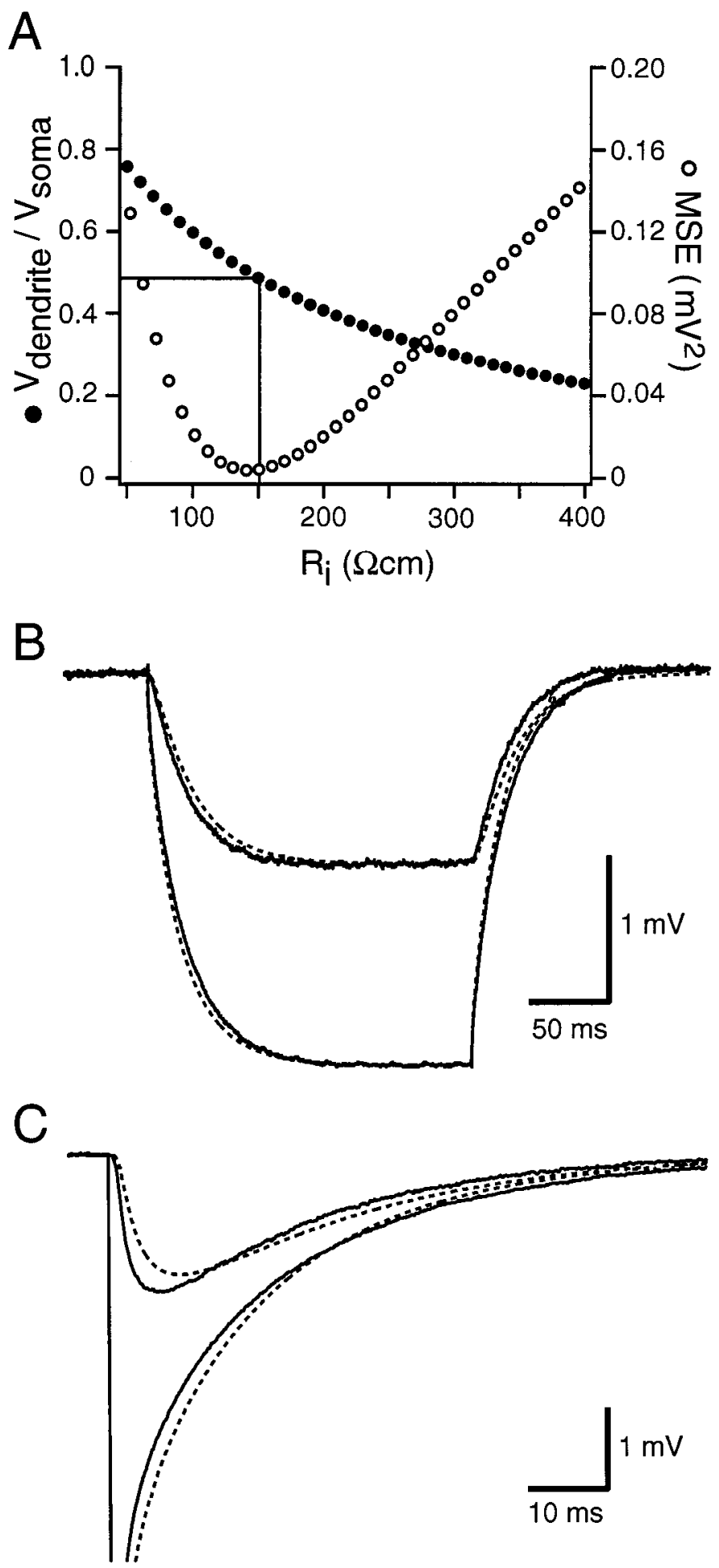

Figure 2. Estimates of $R_{\mathrm{i}}$ models based on steady-state voltage attenuation. $A$, Plot of steady-state attenuation $\left(V_{\text {dendrite }} / V_{\text {soma }} ; \boldsymbol{\bullet}\right)$ and mean squared error $(M S E)$ between the simulated and recorded data $(\bigcirc)$ during long somatic current pulses for the model shown in Figure $1 A$ for values of internal resistivity $\left(R_{\mathrm{i}}\right)$ ranging from 50 to $400 \Omega \mathrm{cm}$. The horizontal line indicates the experimentally recorded steady-state attenuation, showing that this coincides with an $R_{\mathrm{i}}$ value of $151 \Omega \mathrm{cm}$. This $R_{\mathrm{i}}$ value also coincides with the lowest mean squared error. $B$, Recorded (solid lines) and simulated (broken lines) responses to somatic long current pulses $(-50 \mathrm{pA}, 200 \mathrm{msec})$. The simulated data were generated using parameters based on an analysis as shown in Figure $2 A$, for the cell shown in Figure $1 A$. Note that simulated responses decay too slowly in the dendrite and
Furthermore, the simulated dendritic response always rose more slowly and peaked later than the experimental data (Fig. 2C).

In an attempt to improve these fits a direct fitting approach was used (Clements and Redman, 1989). This was achieved by using a search algorithm to find the combination of $R_{\mathrm{m}}, R_{\mathrm{i}}$, and $C_{\mathrm{m}}$ that yielded the smallest mean squared error between the simulated short current pulse response and the experimental data. Initially either the somatic (Fig. 3A, left) or dendritic (Fig. 3B, left) short-pulse data were fit on there own. The best fit to the somatic short-pulse data, however, always gave a poor fit to the dendritic short-pulse data (Fig. 3A, left). Also, as with models based purely on steady-state attenuation (Fig. $2 C$ ), the rising phase of the simulated dendritic short-pulse response was too slow, and the peak occurred too late (Fig. $3 A$ ). The best fit to the dendritic short-pulse data gave poor fits to the somatic short-pulse data (Fig. $3 B$, left). Lower $R_{\mathrm{i}}$ values were provided by direct fits to the dendritic short-pulse data, with $R_{\mathrm{i}}$ ranging from 71 to $92 \Omega \mathrm{cm}$. These models, however, predicted too little steady-state attenuation (Fig. 3B, right). Simultaneously fitting of both the somatic and dendritic short-pulse data produced fits that decayed too rapidly in the soma and too slowly in the dendrite (data not shown, but similar to the fits shown in Fig. 2C).

\section{Simulations with nonuniform $R_{\mathrm{m}}$}

The different time courses of the decay of the somatic and dendritic voltage responses suggest that the membrane properties are nonuniform. Nonuniformities in $R_{\mathrm{i}}$ cannot account for these effects because $R_{\mathrm{i}}$ influences only the initial portion of the response to short current pulses. The faster decay of the dendritic response compared with the soma therefore suggests that $R_{\mathrm{m}}$ or $C_{\mathrm{m}}$ or both are nonuniform and lower in the dendrites than the soma. On the other hand, the rising phase of the dendritic response, which is sensitive to both $R_{\mathrm{i}}$ and $C_{\mathrm{m}}$, was best fit with models with low $R_{\mathrm{i}}$ (Fig. $3 B$ ). As mentioned, these models predicted too little steady-state attenuation, which cannot be accounted for by nonuniformities in $C_{\mathrm{m}}$, because steady-state attenuation is not dependent on $C_{\mathrm{m}}$. This therefore suggests that $R_{\mathrm{m}}$ must be nonuniform and lower in the dendrites than in the soma.

Direct fitting was therefore performed on both the somatic and dendritic data using models in which $R_{\mathrm{m}}$ was made nonuniform (Fig. 3C) (see Materials and Methods). Nonuniform $R_{\mathrm{m}}$ models gave significantly better fits $(p<0.05)$ to the somatic and dendritic short and long current pulse responses for all cells (Fig. 4). The fits with nonuniform $R_{\mathrm{m}}$ models, however, were still imperfect (particularly during the falling phase of the short-pulse responses), which may be because of the way nonuniform $R_{\mathrm{m}}$ was incorporated into these models. Other nonuniform functions were tried (e.g., step, linear) but were either worse or did not improve the fits. Additional experimental data will be needed to determine what refinements might be required to improve these models further.

In cells modeled with nonuniform $R_{\mathrm{m}}$ the best fits obtained with direct fitting to both the somatic and dendritic data predicted

\section{$\leftarrow$}

too quickly in the soma. $C$, Recorded (solid lines) and simulated (broken lines) responses to somatic short current pulses ( $-2 \mathrm{nA}$ for $1 \mathrm{msec})$. Same model as in $B$. Note that the simulated dendritic response peaks too late, and as with the simulated responses to long current pulses $(B)$, the simulated dendritic response decays too slowly, whereas the somatic response decays too quickly. The values of $R_{\mathrm{i}}, C_{\mathrm{m}}$, and $R_{\mathrm{m}}$ in the model shown in $B$ and $C$ were $151 \Omega \mathrm{cm}, 1.3 \mu \mathrm{F} / \mathrm{cm}^{2}$, and $17,981 \Omega \mathrm{cm}^{2}$, respectively. 
A


$\mathrm{B}$

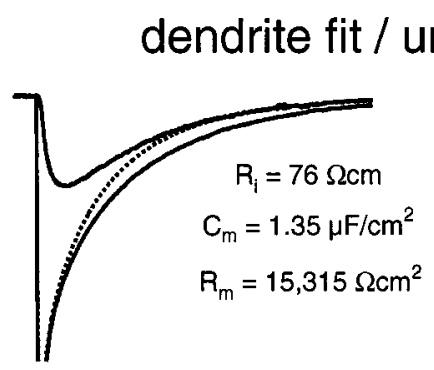

uniform $\mathrm{R}_{\mathrm{m}}$

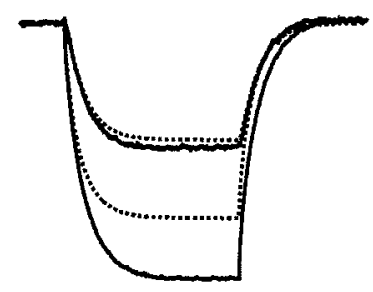

C

\section{both fit / nonuniform $R_{m}$}

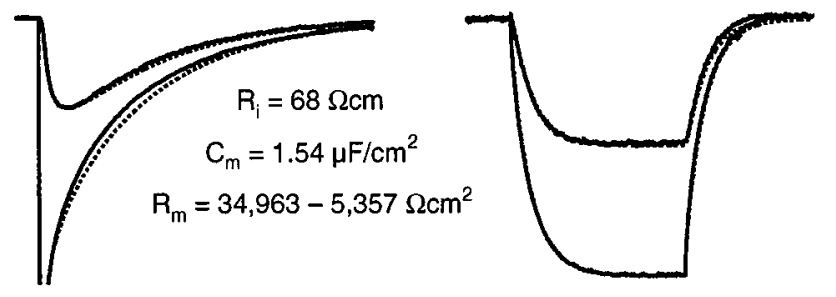

Figure 3. Models with nonuniform $R_{\mathrm{m}}$ provide the best fits to short and long current pulse responses. A, Recorded (solid lines) and simulated (broken lines) responses to somatic short (left, $-2 \mathrm{nA}$ for $1 \mathrm{msec}$ ) and long (right, $-50 \mathrm{pA}, 200 \mathrm{msec}$ ) current pulses using a model derived from a direct fit to the somatic short-pulse data. Same cell as shown in Figure $1 \mathrm{~A}$. The values of $R_{\mathrm{i}}, C_{\mathrm{m}}$, and $R_{\mathrm{m}}$ in this model were $104 \Omega \mathrm{cm}, 1.46 \mu \mathrm{F} / \mathrm{cm}^{2}$, and $18,591 \Omega \mathrm{cm}^{2}$, respectively. Note that the fit to the dendritic shortpulse data are poor. $B$, The same recorded data as in $A$ with superimposed simulations using parameters derived from a direct fit to the dendritic data. Note that the fits to the somatic short-pulse data are poor, and that simulations of the long-pulse response using the same model parameters generated too little steady-state attenuation. The values of $R_{\mathrm{i}}, C_{\mathrm{m}}$, and $R_{\mathrm{m}}$ in this model were $76 \Omega \mathrm{cm}, 1.35 \mu \mathrm{F} / \mathrm{cm}^{2}$, and $15,315 \Omega \mathrm{cm}^{2}$, respectively. $C$, The same recorded data as in $A$ with superimposed simulations derived from a direct fit to both the somatic and dendritic long- and short-pulse data in a model in which $R_{\mathrm{m}}$ was made nonuniform as a function of distance from the soma as described by Equation 1 (see Materials and Methods). The final values of $R_{\mathrm{i}}, R_{\mathrm{m}}$ (soma), $R_{\mathrm{m}}$ (end), $d_{\text {half }}$, steep, and $C_{\mathrm{m}}$ were $68 \Omega \mathrm{cm}, 34,963 \Omega \mathrm{cm}^{2}, 5,357 \Omega \mathrm{cm}^{2}, 406 \mu \mathrm{m}, 50 \mu \mathrm{m}$, and 1.54 $\mu \mathrm{F} / \mathrm{cm}^{2}$, respectively. Similar models with low $R_{\mathrm{i}}$ and nonuniform $R_{\mathrm{m}}$ also provided the best fits of the data from the other two neurons modeled (Figs. $1 B, C$ ).

that $R_{\mathrm{m}}$ was significantly lower in the dendrites than in the soma, with the ratio of $R_{\mathrm{m}}$ (soma) $/ R_{\mathrm{m}}$ (end) ranging from 7 to 36 . These nonuniform $R_{\mathrm{m}}$ models provided better fits to the experimental data because lowering the membrane resistivity in the dendrites makes the dendritic response decay faster than the somatic, and at the same time produces more steady-state voltage attenuation. Although less reliable because of possible errors in the estimation of spine density (see Materials and Methods), $C_{\mathrm{m}}$ values obtained by direct fitting with nonuniform $R_{\mathrm{m}}$ models ranged from 1.1 to

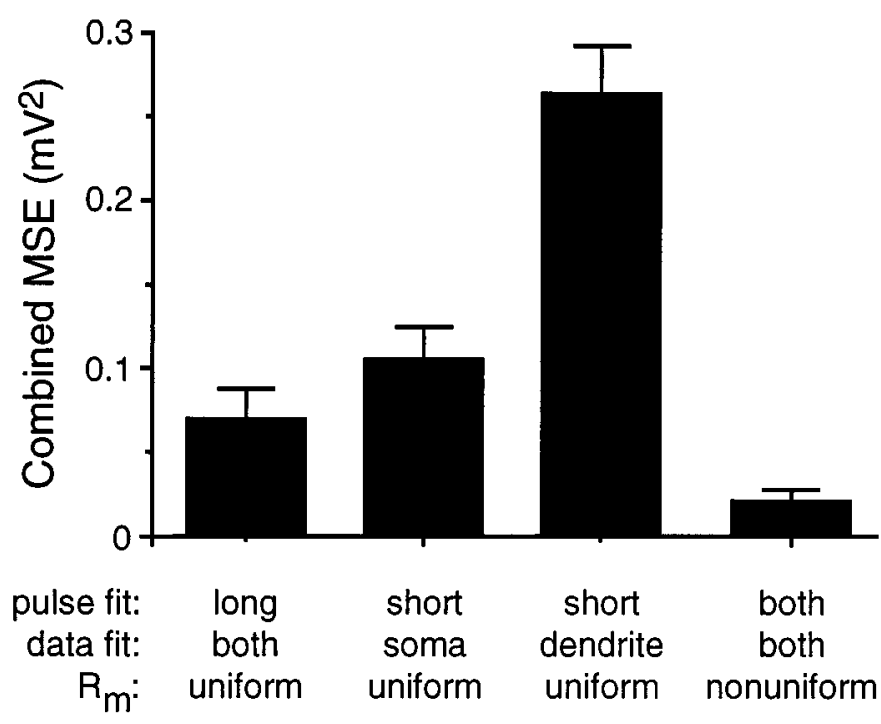

Figure 4. Comparison of the average ( \pm SEM) combined mean squared error $(M S E)$ for fits to the somatic and dendritic long- and short-pulse responses in various models for the three cells analyzed. The text under the histogram indicates (1) whether the long or short current pulses, or both, were used to optimize the fit, (2) whether the somatic or dendritic response, or both, was fit, and (3) whether the model used to generate the fit incorporated uniform or nonuniform $R_{\mathrm{m}}$.

$1.5 \mu \mathrm{F} / \mathrm{cm}^{2} . R_{\mathrm{i}}$ values obtained with nonuniform $R_{\mathrm{m}}$ models ranged from 68 to $105 \Omega \mathrm{cm}$, similar to that predicted from uniform $R_{\mathrm{m}}$ models during direct fitting to only the dendritic short-pulse data (see above). The $R_{\mathrm{i}}$ values obtained with direct fitting are therefore lower than most recent estimates.

\section{Simulations with uniform and nonuniform $I_{\mathrm{h}}$}

In an attempt to simulate the additional steady-state attenuation observed during long-pulse responses under control conditions (Fig. $1 F)$, the sag in the voltage response back to the baseline during somatic hyperpolarizing long current pulses was modeled using the conductance $I_{\mathrm{h}}$ based on previously described parameters (Fig. 5) (Solomon and Nerbonne, 1993a,b). With the passive membrane parameters determined from direct fits with nonuniform $R_{\mathrm{m}}$ (Fig. $3 C$ ), direct fitting was used to incorporate $I_{\mathrm{h}}$ sufficient to simulate the sag observed at the soma. In each of the three modeled neurons, incorporation of $I_{\mathrm{h}}$ at uniform density resulted in simulated dendritic voltage responses that were larger than those observed experimentally (Fig. $5 B$ ). One way to resolve this difference was to incorporate $I_{\mathrm{h}}$ nonuniformly. Direct fitting using models with nonuniform $I_{\mathrm{h}}$ gave much better fits to the experimental control data (Fig. 5C) and predicted that the density of $I_{\mathrm{h}}$ was significantly higher in the distal apical dendrites than in the soma. As with the fits with nonuniform $R_{\mathrm{m}}$, the fits with nonuniform $I_{\mathrm{h}}$ models were still imperfect (particularly during the rising and falling phase of the somatic long current pulse response). This may be attributable to problems in the way $I_{\mathrm{h}}$ was modeled, or to the way nonuniform $I_{\mathrm{h}}$ was distributed in these models. Alternatively, the increased steady-state attenuation in control compared with that seen in uniform $I_{\mathrm{h}}$ models may be attributable to $\mathrm{CsCl}$-sensitive conductances other than $I_{\mathrm{h}}$, which are also open at rest (Spruston and Johnston, 1992) and present at a higher density in the distal apical dendrites than in the soma and proximal dendritic regions. As with nonuniform $R_{\mathrm{m}}$ models, additional experimental data will be required to determine what 
A no $I_{h}$

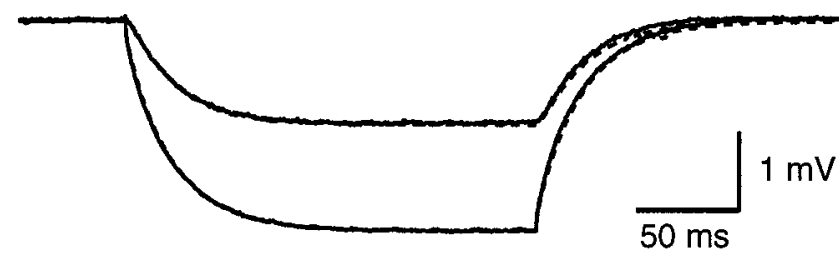

B

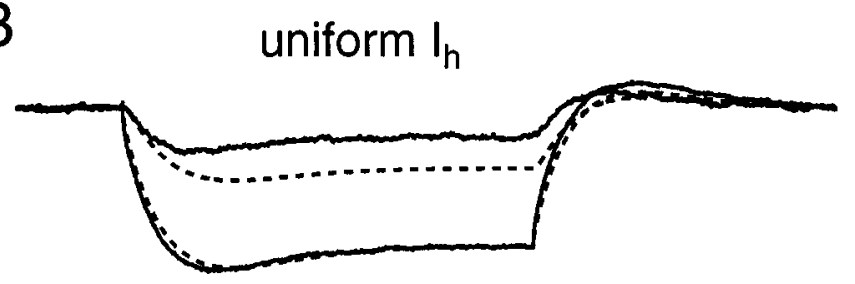

C

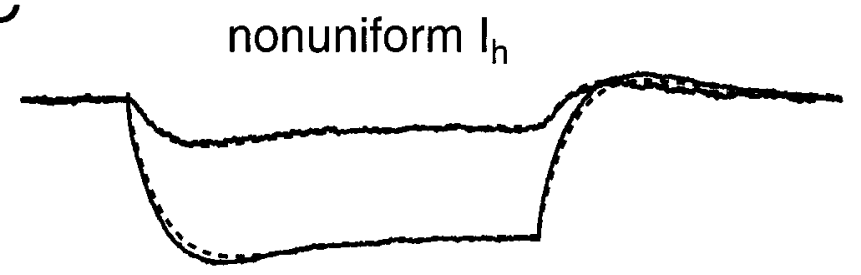

Figure 5. A nonuniform distribution of Cs-sensitive conductances is required to fit the steady-state attenuation observed in control conditions. $A$, Recorded (solid lines; in the presence of $\mathrm{CsCl}$ ) and simulated (broken lines) long-pulse responses. Data from the cell shown in Figure $1 A$, modeled with nonuniform $R_{\mathrm{m}}$ as in Figure $3 C . B$, Addition of a uniform density of $I_{\mathrm{h}}$ to the model shown in $A$ simulates the sag in the somatic control response but predicts too little steady-state voltage attenuation. The values of $g_{\mathrm{h}}$ and $R$ in this model were $0.0282 \mathrm{mS} / \mathrm{cm}^{2}$ and 7.83 respectively. $C$, Addition of a nonuniform distribution of $I_{\mathrm{h}}$ to the model shown in $A$, with a higher density in the distal apical dendrites, adequately simulates the experimentally observed control steady-state attenuation. The density of $I_{\mathrm{h}}$ in different compartments $\left(g_{\mathrm{h}}\right)$ ranged from low to high as a function of distance from the soma as described by Equation 2 (see Materials and Methods), with final values of $g_{\mathrm{h}}$ (soma), $g_{\mathrm{h}}$ (end), $d_{\text {half }}$, steep, and $R$ of $0.020 \mathrm{mS} / \mathrm{cm}^{2}, 20 \mathrm{mS} / \mathrm{cm}^{2}, 439 \mu \mathrm{m}, 50 \mu \mathrm{m}$, and 1.27 , respectively. Similar models with nonuniform $I_{\mathrm{h}}$ also provided the best fits of the data from the other two neurons modeled (Figs. 1B,C).

refinements will be necessary to further improve the fits to nonuniform $I_{\mathrm{h}}$ models.

\section{Attenuation of synaptic potentials}

Simulations of synaptic potentials in models with nonuniform membrane properties suggested an independent experimental test of some of the conclusions from the modeling described above. In models with nonuniform $R_{\mathrm{m}}$ and $I_{\mathrm{h}}$, crossover of the somatic and dendritic EPSPs was observed, with the dendritic voltage becoming smaller than the somatic voltage during the decay phase of the dendritic EPSP (Fig. 6A, left). Although not as dramatic as in models that also included nonuniform $I_{\mathrm{h}}$, models with only nonuniform $R_{\mathrm{m}}$ also showed crossover of the dendritic and somatic EPSPs during their decay (Fig. $6 B$, left). Such behavior was never observed in models with uniform $R_{\mathrm{m}}$ and no $I_{\mathrm{h}}$ (data not shown). In models with uniform $R_{\mathrm{m}}$ and uniform $I_{\mathrm{h}}$, some crossover was observed, but the extent of this crossover was
A model

experiment
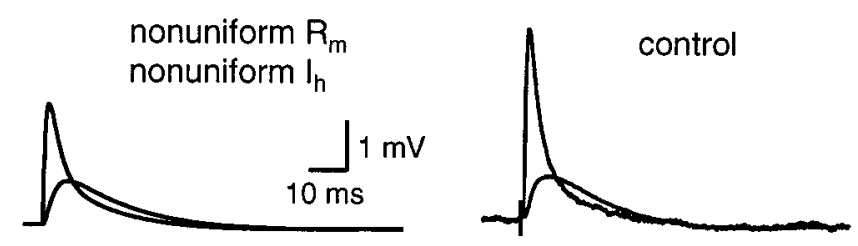

B

nonuniform $\mathrm{R}_{\mathrm{m}}$ no $I_{h}$
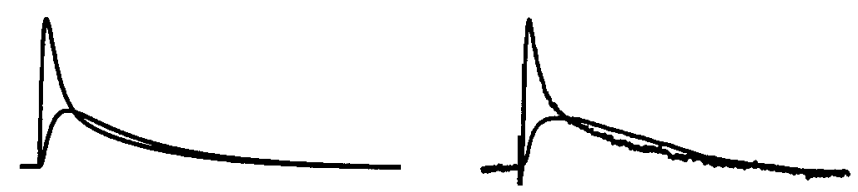

C
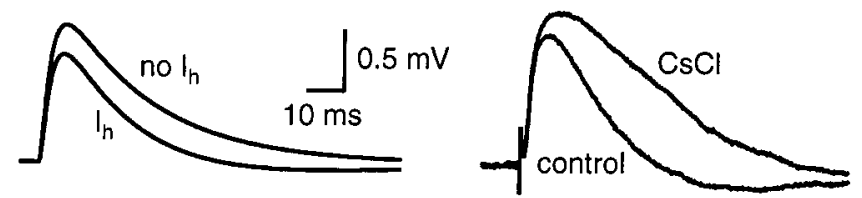

Figure 6. Effects of resting conductances on EPSP attenuation and decay. A, Left, simulated dendritic (larger response, $403 \mu \mathrm{m}$ from the soma) and somatic (smaller response) EPSPs using nonuniform $R_{\mathrm{m}}$ and $I_{\mathrm{h}}$ in the model shown in Figure $5 C$. Simulated EPSPs were generated with a transient, $5 \mathrm{nS}$ conductance increase distributed at five sites between 350 and $450 \mu \mathrm{m}$ from the soma. Right, experimental data of EPSPs evoked by extracellular synaptic stimulation close to the dendritic recording pipette (400 $\mu \mathrm{m}$ from the soma) under control conditions (not the same cell as simulated). B, Left, simulations of EPSPs with nonuniform $R_{\mathrm{m}}$ and no $I_{\mathrm{h}}$. Right, experimental EPSPs recorded in the presence of $5 \mathrm{~mm} \mathrm{CsCl}$ (same cell as in $A$ ). Note the crossover of the somatic and dendritic EPSPs in both $A$ and $B$. Calibration same as in $A$. $C$, Comparison of simulated (left) and experimentally recorded (right) somatic EPSPs under the same conditions as in $A$ and $B$. Note the additional attenuation and accelerated decay produced by resting Cs-sensitive conductances such as $I_{\mathrm{h}}$.

so small that it would be expected to be buried in the noise of an experimental recording (data not shown).

Experimental synaptic stimulation close to the dendritic recording electrode under control conditions (Fig. 6A, right) and in the presence of $5 \mathrm{~mm} \mathrm{CsCl}$ (Fig. $6 B$, right) produced EPSPs that behaved in a way similar to that predicted by models with nonuniform channel distributions, with dendritically recorded EPSPs crossing over the somatic EPSPs during the decay phase and with the extent of this crossover depending on whether $\mathrm{CsCl}$ was present. The similarity between these experimental results and the behavior predicted by the different models provides further experimental support for the conclusion that both Cs-sensitive and Cs-insensitive conductances are distributed nonuniformly and are present at the highest density in the distal apical dendrites of neocortical pyramidal neurons.

Figure 6 also demonstrates how resting Cs-sensitive conductances affect the attenuation of EPSPs generated in the main apical dendrite $\sim 400 \mu \mathrm{m}$ from the soma. For evoked events in the presence of $5 \mathrm{~mm} \mathrm{CsCl,} \mathrm{somatic} \mathrm{EPSP} \mathrm{peak} \mathrm{amplitude} \mathrm{was}$ 
increased on average by $15 \pm 4 \%$ and integral by $99 \pm 14 \%$ relative to control (Fig. $5 C$, right) $(n=5)$. Similar effects were seen at the soma in simulations when models were compared with and without $I_{\mathrm{h}}$ (Fig. 5C, left). These findings show that resting Cs-sensitive conductances not only increase steady-state voltage attenuation (Fig. $1 F$ ) but also reduce EPSP amplitude and duration.

\section{DISCUSSION}

\section{Passive membrane properties}

One of the main findings of this study is that the intracellular resistivity $\left(R_{\mathrm{i}}\right)$ of neocortical layer 5 pyramidal neurons is approximately 70 to $100 \Omega \mathrm{cm}$. This is substantially lower than most recent estimates of $R_{\mathrm{i}}$ (Shelton, 1985; Stratford et al., 1989; Cauller and Connors, 1992; Fromherz and Muller, 1994; Major et al., 1994; Rapp et al., 1994; Thurbon et al., 1994; Bekkers and Stevens, 1996; Meyer et al., 1997) and more in line with the value used by early investigators of the passive properties of CNS neurons (Coombs et al., 1959; Rall, 1959; Lux et al., 1970; Barrett and Crill, 1974), and that recently obtained in spinal motoneurons (Thurbon et al., 1998)

It has been recognized for some time that better estimates of membrane properties such as $R_{\mathrm{i}}$ will require voltage measurement at two points (or more) on the same neuron, especially if membrane properties are nonuniform (Holmes and Rall, 1992). It is precisely for this reason that the present study was undertaken using simultaneous somatic and dendritic recording. The vast majority of previous estimates of membrane properties of neurons in the CNS have relied on the voltage- or current-response measured with a single electrode at the soma (see introductory remarks). Although theoretically direct fitting of the response recorded at a single location should be able to be used to accurately measure $R_{\mathrm{m}}, C_{\mathrm{m}}$, and $R_{\mathrm{i}}$, once one or more of these parameters are made nonuniform the solution obtained becomes nonunique (Holmes and Rall, 1992). That this is the case can be seen from the fits to either the somatic (Fig. $3 A$ ) or dendritic (Fig. $3 B$ ) short current pulse data. In both cases the fits of the limited data set were perfect, yet the predicted model poorly described all of the experimental data.

The fact that our method of determining $R_{\mathrm{i}}$ purely from the extent of steady-state voltage attenuation during a long somatic current pulse gave higher values of $R_{\mathrm{i}}$ than those obtained from direct fitting using nonuniform $R_{\mathrm{m}}$ models is presumably because this simpler approach assumed that $R_{\mathrm{m}}$ was uniform. As can be seen in Figure $3 C$, the lower values of $R_{\mathrm{i}}$ combined with nonuniform $R_{\mathrm{m}}$ (lower in the distal dendrites) not only predicted accurately the observed steady-state attenuation, but also predicted more accurately the time course of the response to both somatic short and long current pulses (compare Fig. 2B, $C$ with Fig. 3C).

The possibility that the measured passive properties were affected by cytoplasmic dialysis of the cell with the whole-cell recording solution is unlikely because the control somatic input resistance was similar during whole-cell and perforated-patch recording. Furthermore, the extent of sag and the increase in input resistance in $\mathrm{CsCl}$ were similar during whole-cell and perforated-patch recording, suggesting that whole-cell recording also has little effect on Cs-sensitive conductances. That similar results were observed with perforated-patch and whole-cell recording suggests that the intracellular components that make up $R_{\mathrm{i}}$ consist of immobile molecules and elements, such as organelles and cytoskeletal proteins.

\section{Nonuniformly distributed resting conductances}

A second important finding from this study is that resting conductances (both Cs-sensitive and Cs-insensitive) are present at higher densities in the distal apical dendrites in neocortical pyramidal neurons. When the Cs-sensitive conductance $I_{\mathrm{h}}$ was incorporated into our passive model, we found that a uniform channel distribution resulted in less steady-state attenuation than we had observed experimentally (Fig. 5B). The simplest explanation of this finding is that Cs-sensitive conductances are distributed nonuniformly and at a higher density in the distal apical dendrites. This conclusion was supported further by the finding that predicted "crossover" during the decay of somatic and dendritic EPSPs was observed experimentally, and that this crossover was reduced by the application of $\mathrm{CsCl}$ (Fig. 6). This crossover is unlikely to be caused by activation of other voltage-activated conductances, because previous studies have shown that there is little contribution of voltage-activated $\mathrm{Na}^{+}$or $\mathrm{Ca}^{2+}$ conductances to small ( $<5 \mathrm{mV}$ at the soma) EPSPs generated in the main apical dendrite of layer $\mathrm{V}$ pyramidal neurons (Stuart and Sakmann, 1995).

Together, these results provide strong evidence that Cssensitive conductances are present at a higher density in the distal apical dendrites. Whether this nonuniform channel distribution is attributable to differences in the somatic and dendritic density of $I_{\mathrm{h}}$ or is rather due to differences in the density of other Cs-sensitive conductances remains to be determined. Ultimately, the exact distributions of resting conductances will require direct approaches such as patch-clamp recordings from isolated dendritic and somatic patches and possibly analysis of channel distribution by antibody binding studies.

A recent study in hippocampal pyramidal neurons has found that the density of a transient, A-type potassium conductance is also higher in the distal apical dendrites than at the soma and more proximal regions (Hoffman et al., 1997). These authors show that this has the effect of dampening dendritic excitability in hippocampal dendrites. Although the higher density of Cssensitive conductances in the distal apical dendrites of layer 5 pyramidal neurons is unlikely to be attributable to the presence of a high density of A-type potassium channels, which are relatively insensitive to external Cs (Hille, 1994), dendritic Cs-sensitive conductances such as $I_{\mathrm{h}}$ would be expected to have a similar effect on dendritic excitability. The predicted high density of $I_{\mathrm{h}}$ in the apical dendrite of neocortical layer 5 pyramidal neurons may also play an important role in regulating the extent with which regenerative events generated in the distal apical dendrites (Schiller et al., 1997; Stuart et al., 1997a) propagate to the soma and axon (Schwindt and Crill, 1997).

\section{EPSP attenuation}

The expected attenuation of EPSPs generated in the most distal apical tuft dendrites ( $>1000 \mu \mathrm{m}$ from the soma) is shown in Figure 7 using models of a layer 5 pyramidal neurons having either uniform or nonuniform $R_{\mathrm{m}}$, with uniform and nonuniform $I_{\mathrm{h}}$. In all models, dramatic attenuation (greater than 100-fold) of EPSP peak amplitude occurs as these EPSPs propagate from the distal apical dendrites toward the soma. Comparison of uniform and nonuniform $R_{\mathrm{m}}$ models without $I_{\mathrm{h}}$ (Fig. $7 B, C$ ) reveals that the nonuniform $R_{\mathrm{m}}$ model results in a slightly smaller EPSP at its dendritic site of generation, whereas the EPSP at the soma is 


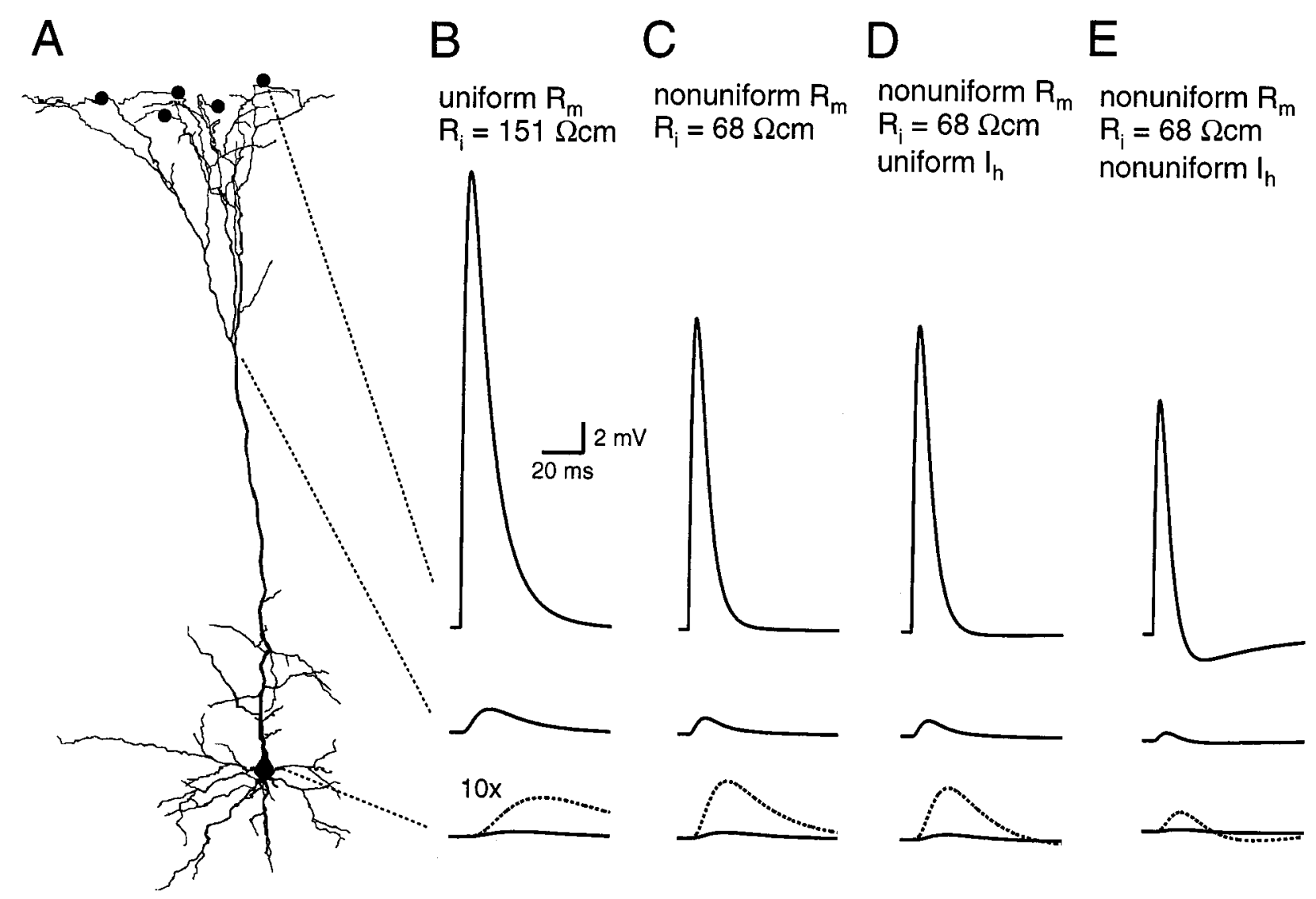

Figure 7. Attenuation of distally generated EPSPs in different models. $A$, Representation of the dendritic morphology of the neuron modeled (same cell as in Fig. $1 A$ ). The distance to the middle recording location in this simulation is $596 \mu \mathrm{m}$ from the soma. The sites of simulated synaptic inputs are indicated by the black dots. $B-E$, Simulated EPSPs recorded in four different models at three separate locations: a distal apical tuft branch near the site of one synaptic input $(\sim 1000 \mu \mathrm{m}$ from the soma), a more proximal apical dendritic location just on the somatic side of the first major apical branch point (596 $\mu \mathrm{m}$ from the soma), and at the soma. The somatic EPSPs are also shown amplified 10 times (broken lines). EPSPs were simulated by a transient, $5 \mathrm{nS}$ conductance increase in the distal apical dendritic tuft ( $>1000 \mu \mathrm{m}$ from the soma), which was distributed over the five sites indicated in $A$ (i.e., each individual synapse had a conductance of $1 \mathrm{nS}$ ). The different models used were either $B$, a high $R_{\mathrm{i}}$, uniform $R_{\mathrm{m}}$ model (as in Fig. 2); $C$, a lower $R_{\mathrm{i}}$, nonuniform $R_{\mathrm{m}}$ model (as in Fig. $3 C$ ); $D$, a lower $R_{\mathrm{i}}$, nonuniform $R_{\mathrm{m}}$, uniform $I_{\mathrm{h}}$ model (as in Fig. $5 B$ ); or $E$, a lower $R_{\mathrm{i}}$, nonuniform $R_{\mathrm{m}}$, nonuniform $I_{\mathrm{h}}$ model (as in Fig. $5 C$ ).

actually larger (Fig. 7C). In contrast, in models in which $I_{\mathrm{h}}$ was nonuniform and higher in the distal dendrites (Fig. $7 E$ ), the size of the distal EPSP at its site of generation was smaller, and attenuation of this EPSP to the soma was significantly greater than in the other models. Attenuation of large distal EPSPs could be reduced if they activate dendritic voltage-activated $\mathrm{Na}^{+}$or $\mathrm{Ca}^{2+}$ conductances, as can be the case under some conditions (Schiller et al., 1997; Stuart et al., 1997a).

In contrast to their effect on distal EPSPs, Cs-sensitive conductances had little effect at the site of origin on the amplitude of EPSPs generated in main apical dendrite $\sim 400$ $\mu \mathrm{m}$ from the soma, and they reduced the amplitude of these EPSPs at the soma by on average only $15 \%$ (Fig. 6). This difference presumably comes about because $I_{\mathrm{h}}$ has the greatest effect on EPSPs generated at locations where the density of $I_{\mathrm{h}}$ is highest.

The primary effect of resting Cs-sensitive conductances was on EPSP integral rather than peak. For EPSPs generated relatively proximally in the apical dendrite (400 $\mu \mathrm{m}$ from the soma), application of $\mathrm{CsCl}$ slowed the EPSP decay, almost doubling the evoked EPSP integral at the soma relative to control (Fig. 6). The effect on more distally generated EPSPs was even more dramatic, with a threefold difference in somatic EPSP integral in models with and without nonuniform $I_{\mathrm{h}}$ (Fig. $7 E$ ). As a consequence, these Cs-sensitive conductances significantly decrease the synaptic charge that reaches the soma and axon. Cs-sensitive conductances also decrease somatic EPSP duration and therefore reduce the time interval over which temporal summation of synaptic events can occur. Other studies have also observed that agents that block $I_{\mathrm{h}}$ lead largely to an increase in EPSP duration (Nicoll et al., 1993).

Together, these results show that Cs-sensitive and Csinsensitive nonuniform conductances generate a "leaky" apical dendrite, increasing voltage attenuation along the apical dendrite of neocortical layer 5 pyramidal neurons despite a relatively low internal resistivity. The major effect of this leaky apical dendrite is to reduce somatic EPSP integral and duration, thereby reducing the time available for temporal summation of synaptic events at the soma and axon. In addition, the effect that these resting conductances have on EPSP amplitude and integral, both at the site of EPSP generation and at the soma, is dependent on EPSP location and is greatest for more distal EPSPs. Modulation of resting conductances such as $I_{\mathrm{h}}$ by receptor-mediated activation 
of intracellular signaling cascades (Benson et al., 1988; Nicoll, 1988; McCormick and Pape, 1990) may therefore provide a powerful way by which cortical pyramidal neurons control synaptic integration, particularly for synaptic inputs onto the distal apical dendrites.

\section{REFERENCES}

Barrett JN, Crill WE (1974) Specific membrane properties of cat motoneurones. J Physiol (Lond) 239:301-324.

Bekkers JM, Stevens CF (1996) Cable properties of cultured hippocampal neurons determined from sucrose-evoked miniature EPSPs. J Neurophysiol 75:1250-1255.

Benson DM, Blitzer RD, Landau EM (1988) An analysis of the depolarization produced in guinea-pig hippocampus by cholinergic receptor stimulation. J Physiol (Lond) 404:479-496.

Brent RP (1973) Algorithms for minimization without derivatives. Englewood Cliffs, NJ: Prentice-Hall.

Brown TH, Perkel DH, Norris JC, Peacock JH (1981) Electrotonic structure and specific membrane properties of mouse dorsal root ganglion neurons. J Neurophysiol 45:1-15.

Bush PC, Sejnowski TJ (1993) Reduced compartmental models of neocortical pyramidal cells. J Neurosci Methods 46:159-166.

Cauller LJ, Connors BW (1992) Functions of very distal dendrites: experimental and computational studies of layer I synapses on neocortical pyramidal cells. In: Single neuron computation (McKenna T, Davis J, Zornetzer SF, eds), pp 199-229. Boston: Academic.

Clements JD, Redman SJ (1989) Cable properties of cat spinal motoneurones measured by combining voltage clamp, current clamp and intracellular staining. J Physiol (Lond) 409:63-87.

Coombs JS, Curtis DR, Eccles JC (1959) The electrical constants of the motoneuronal membrane. J Physiol (Lond) 145:505-528.

Durand D, Carlen PL, Gurevich N, Ho A, Kunov HJ (1983) Electrotonic parameters of rat dentate granule cells measured using short current pulses and HRP staining. J Neurophysiol 50:1080-1097.

Fromherz P, Muller CO (1994) Cable properties of a straight neurite of a leech neuron probed by a voltage-sensitive dye. Proc Natl Acad Sci USA 91:4604-4608.

Hille B (1994) Ion channels of excitable membranes. Sunderland, MA: Sinauer.

Hines ML, Carnevale NT (1997) The NEURON simulation environment. Neural Comput 9:1179-1209.

Hoffman DA, Magee JC, Colbert CM, Johnston D (1997) $\mathrm{K}^{+}$channel regulation of signal propagation in dendrites of hippocampal pyramidal neurons. Nature 387:869-875.

Holmes RW (1989) The role of dendritic diameter in maximizing the effectiveness of synaptic inputs. Brain Res 478:127-137.

Holmes WR, Rall W (1992) Estimating the electrotonic structure of neurons with compartmental models. J Neurophysiol 68:1438-1452.

Iansek R, Redman SJ (1973) An analysis of the cable properties of spinal motoneurones using a brief intracellular current pulse. J Physiol (Lond) 234:613-636.

Kyrozis A, Reichling DB (1995) Perforated-patch recording with gramicidin avoids artifactual changes in intracellular chloride concentration. J Neurosci Methods 57:27-35.

Larkman AU (1991) Dendritic morphology of pyramidal neurones of the visual cortex of the rat: III. Spine distributions. J Comp Neurol 306:332-343.

Llinas R, Sugimori M (1980a) Electrophysiological properties of in vitro Purkinje cell dendrites in mammalian cerebellar slices. J Physiol (Lond) 305:197-213.

Llinas R, Sugimori M (1980b) Electrophysiological properties of in vitro Purkinje cell somata in mammalian cerebellar slices. J Physiol (Lond) 305:171-195.

Lux HD, Schubert P, Kreutzberg GW (1970) Direct matching of morphological and electrophysiological data in motoneurons. In: Excitatory synaptic mechanisms (Andersen P, Jansen JKS, eds), pp. 189-198. Oslo: Universitetsforlaget.

Major G, Larkman AU, Jonas P, Sakmann B, Jack JJB (1994) Detailed passive cable models of whole-cell recorded CA3 pyramidal neurons in rat hippocampal slices. J Neurosci 14:4613-4638.

McCormick DA, Pape HC (1990) Noradrenergic and serotonergic modulation of a hyperpolarization-activated cation current in thalamic relay neurones. J Physiol (Lond) 431:319-342.

Meyer E, Muller CO, Fromherz P (1997) Cable properties of dendrites in hippocampal neurons of the rat mapped by a voltage-sensitive dye. Eur J Neurosci 9:778-785.

Nicoll A, Larkman A, Blakemore C (1993) Modulation of EPSP shape and efficacy by intrinsic membrane conductances in rat neocortical pyramidal neurons in vitro. J Physiol (Lond) 468:693-710.

Nicoll RA (1988) The coupling of neurotransmitter receptors to ion channels in the brain. Science 241:545-551.

Rall W (1959) Branching dendritic trees and motoneuron membrane resistivity. Exp Neurol 1:491-527.

Rapp M, Segev I, Yarom Y (1994) Physiology, morphology and detailed passive models of guinea-pig cerebellar Purkinje cells. J Physiol (Lond) 474:101-118.

Schiller J, Schiller Y, Stuart G, Sakmann B (1997) Calcium action potentials restricted to distal apical dendrites of rat neocortical pyramidal neurons. J Physiol (Lond) 605-616.

Schwindt PC, Crill WE (1997) Modification of current transmitted from apical dendrite to soma by blockade of voltage- and $\mathrm{Ca}^{2+}$-dependent conductances in rat neocortical pyramidal neurons. J Neurophysiol 78:187-198.

Shelton DP (1985) Membrane resistivity estimated for the Purkinje neuron by means of a passive computer model. Neuroscience 14:111-131.

Solomon JS, Nerbonne JM (1993a) Hyperpolarization-activated currents in isolated superior colliculus-projecting neurons from rat visual cortex. J Physiol (Lond) 462:393-420.

Solomon JS, Nerbonne JM (1993b) Two kinetically distinct components of hyperpolarization-activated current in rat superior colliculusprojecting neurons. J Physiol (Lond) 469:291-313.

Spain WP, Schwindt PC, Crill WE (1987) Anomalous rectification in neurons from cat sensorimotor cortex in vitro. J. Neurophysiol 57: $1555-1576$.

Spruston N, Johnston D (1992) Perforated patch-clamp analysis of the passive membrane properties of three classes of hippocampal neurons. J Neurophysiol 67:508-529.

Stratford K, Mason A, Larkman A, Major G, Jack J (1989) The modelling of pyramidal neurones in the visual cortex. In: The computing neurone (Durbin R, Miall C, Mitchison G, eds), pp 298-321. Reading, England: Addison-Wesley.

Stuart G, Sakmann B (1995) Amplification of EPSPs by axosomatic sodium channels in neocortical pyramidal neurons. Neuron 15: 1065-1077.

Stuart G, Schiller J, Sakmann B (1997a) Action potential initiation and propagation in rat neocortical pyramidal neurons. J Physiol (Lond) 617-632.

Stuart G, Spruston N, Sakmann B, Häusser M (1997b) Action potential initiation and backpropagation in neurons of the mammalian central nervous system. Trends Neurosci 20:125-131.

Stuart GJ, Sakmann B (1994) Active propagation of somatic action potentials into neocortical pyramidal cell dendrites. Nature 367:69-72.

Stuart GJ, Dodt H-U, Sakmann B (1993) Patch-clamp recordings from the soma and dendrites of neurons in brain slices using infrared video microscopy. Pflügers Arch 423:511-518.

Thurbon D, Field A, Redman S (1994) Electrotonic profiles of interneurons in stratum pyramidale of the CA1 region of rat hippocampus. J Neurophysiol 71:1948-1958.

Thurbon D, Lusher H-R, Hofstetter T, Redman S (1998) Passive electrical properties of ventral horn neurons in rat spinal cord. J Neurophysiol, in press. 